\title{
Minimum Acceptable Face Velocities of Laboratory Fume Hoods and Guidelines for Their Classification
}
N. E. Bolton
W. E. Porter
S. P. Alcorn
W. S. Everett
J. B. Hunt
J. F. Morehead
H. F. Higdon 


\section{DISCLAIMER}

This report was prepared as an account of work sponsored by an agency of the United States Government. Neither the United States Government nor any agency Thereof, nor any of their employees, makes any warranty, express or implied, or assumes any legal liability or responsibility for the accuracy, completeness, or usefulness of any information, apparatus, product, or process disclosed, or represents that its use would not infringe privately owned rights. Reference herein to any specific commercial product, process, or service by trade name, trademark, manufacturer, or otherwise does not necessarily constitute or imply its endorsement, recommendation, or favoring by the United States Government or any agency thereof. The views and opinions of authors expressed herein do not necessarily state or reflect those of the United States Government or any agency thereof. 


\section{DISCLAIMER}

Portions of this document may be illegible in electronic image products. Images are produced from the best available original document. 
Printed in the United States of America. Available from National Technical Information Service

U.S. Department of Commerce 5285 Port Royal Road, Springfield, Virginia 22161

Price: Printed Copy Microfiche

This report was prepared as an account of work sponsored by an agency of the United States Government. Neither the United States Government nor any agency thereof, nor any of their employees, contractors, subcontractors, or their employees, makes any warranty, express or implied, nor assumes any logal liability or responsibility for any third party's use or the results of such use of any information, apparatus, product or process disclosed in this report, nor represents that its use by such third party would no? infringe privately owned rights. 
ORNL/TM- 6400

Contract No. W-7405-eng-26

HEALTH DIVISION

MINIMUM ACCEPTABLE FACE VELOCITIES OF LABORATORY FUME HOODS AND GUIDELINES FOR THEIR CLASSIFICATION

* - Y-12 Plant

- ORGDP Plant
N. E. Bolton
W. E. Porter
S. P. Alcorn
W. S. Everett*
J. B. Hunt*
J. F. Morehead*.
H. F. Higdon?

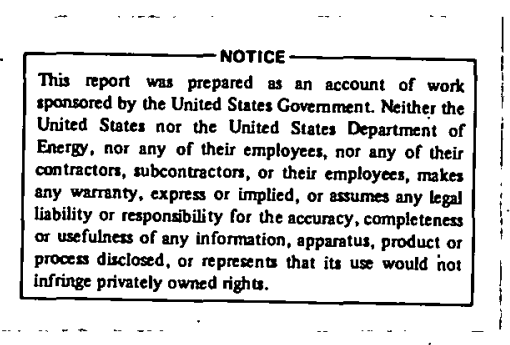

Date Published - June, 1978

NOTICE This document contains information of a preliminary nature. It is subject to revision or correction and therefore does not represent a final report.

\footnotetext{
UAK KIDGE NATIONAL LABORATORY

Oak Ridge, Tennessee 37830

operated by

UNION CARBIDE CORPORATION

for the

DEPARTMENT OF ENERGY
} 


\section{THIS PAGE \\ WAS INTENTIONALLY \\ LEFT BLANK}




\section{ABSTRACT}

This report summarizes the data developed to support the requirement of a $100 \mathrm{LFM}$ minimum face velocity requirement for laboratory fume hoods. Also included is a description of the Y-12 test hood as well as guidelines for a hood classification scheme. 


\section{INTRODUCTION}

In February 1974, a joint study was undertaken by ORNL, ORGDP and Y-12 to determine the adequacy of laboratory (fume) hoods. The purpose of this report is to summarize the data which has been collected to define adequate face velocities for specific hood classifications.

In March 1974, recommended guidelines for the classification of laboratory hoods was issued by industrial hygiene representatives of the four UCC-ND plants (see Appendix $A$ ). It was recognized that utilization of any classification scheme would, in actual practice, be difficult to administer effectively. In addition, professional judgment would be required to review special situations and make exceptions to the established classifications.

To reconcile difficulties a multiple approach was taken. A test hood was purchased and installed in the $\mathrm{Y}-12$ plant to determine adequacy of given flow rates for given concentrations of toxic chemicals. A complete description of this installation is given in Appendix B. In addition, breathing zone sampling was conducted for a significant number of actual operations involving a variety of toxic chemicals. Also, synthetic situations were employed using multiple concentration factors of TLV's for carbon monoxide and perchloroethylene. A complete description of each of these activities is presented.

\section{DISCUSSION}

I. Laboratory Test Hood $(y-12)$.

Data on three test chemicals are given in Table 1. Because of the generation method used, it was not possible to determine the precise concentration inside the hood. Concentrations generated inside the hood were therefore assigned a greater than or equal concentration.

From these data it is apparent that a flow rate of 100 LFM (with 408 sash opening) will prevent the concentration in the breathing zone from exceeding the TLV on carbon tetrachloride and perchloroethylene. It is also apparent that handling Freon 113 under most conditions would not present a problem.

In actual practice, prófessional judgment would be applied to situations involving carbon tetrachloride and a minimum flow rate of 125 LFM would probably be recommended. Regardless of the flow rate 
on a given hood, breathing zone sampling would be required to insure the adequacy of the ventilation system in preventing exposure to materials as toxic as $\mathrm{CCL}_{4}$, Beryllium, carcinogens, etc.

\section{Breathing Zone Measurements}

A. ORNL Data. The data in Table 2 represent workroom air samples taken in the breathing zone of workers during actual operations which were being performed in laboratory hoods. In all cases these results reflect "live" workroom conditions; that is, none were synthetic or mock-up to provide a sample. Concentrations of contaminants are in parts per million parts air by volume (ppm), with the exception of those for mercury and beryllium which, of course, are expressed in milligrams per cubic meter of air $\left(\mathrm{mg} / \mathrm{m}^{3}\right)$. Threshold Limit Values (TLV's) are expressed in the same units. In the operations sampled none of the contaminants used exceeded or even approached the TLV concentrations. From these data, it seems reasonable to conclude that a face velocity of 100 LFM (with 408 sash opening) is adequate to control breathing zone concentrations of most contaminants used in ORNI laboratory hood operations.

B. Y-12 Data. As with the ORNL data (previous paragraph), data have been collected and summarized in. Tables 3 and 4 for Y-12 operations. These data also were collected during actual operations and reflect the concentrations to which employees would have been exposed had respiratory protection not been used. Data in Table 3 summarizes the results of 221 breathing zone samples for beryllium and 381 breathing zone samples for uranium. Since the operations involve very small quantities of the materials, the potential for exposure is very low. The air flows versus concentrations detected suggest protection can be achieved with fairly low face velocities in operations of thie type. Howcrer, opcrations of this type are subjecl tu day-today changes. Hence, professional judgement would dictate 100 LFM as the minimum face velocity. for these operations.

Data in Table 4 were secured during operations involving large quantities of beryllium powder of fairly small particle size. These breathing zone samples were secured on employees using respiratory 
protection and clearly show that for these operations $i 34$ and 124

LFM would not have been adequate to protect the employees from concentrations in excess of the TLV.

c. ORGDP Data. The data in Table 5 represent ORGDP air samples secured in the breathing zone of workers during actual operations being performed involving the contaminant identified. Except as indicated the face velocities were measured with the sash full open. Consequently, all face velocities would increase to $\geq 100$ LFM with a 408 sash opening. Here again it is apparent that under the conditions of the tests, 100 LFM face velocity is adequate to prevent concentration approaching the TLV in the breathing zone.

\section{Synthetic Situations for Breathing zone Sampling}

At ORNL as an alternative to performing breathing zone sampling on every employee potentially exposed to a toxic chemical, a system was devised using carbon monoxide as the test gas. Pure carbon monoxide gas was metered in at the air foil on a series of hoods in quantities sufficient to produce $10 \mathrm{TLV}$ 's of $\mathrm{CO}(500 \mathrm{ppm})$. The individual performing the test then went through a series of motions designed to simulate actual work movements. Breathing zone concentrations were determined during these activities. These tests were conducted on 350 laboratory hoods in the 4500 complex. Some 1000 to 1200 determinations were made during this investigation for carbon monoxide in the breathing zone.

The highest concentration detected was $<5 \mathrm{ppm}$ or $<10 \% \mathrm{TLV}$. (Note: $5 \mathrm{ppm}$ is considered the lower limit of detection for the MSA carbon monoxide detector tube.) These data suggest that air flows of 100 LFM or greater (at a 40 sash opening) do, in fact, protect the employees from exposure to carbon monoxide in excess of the TLV.

Similar studies were conducted by $\mathrm{Y}-12$ using perchloroethylene. Their data also showed that adequate protection is afforded the employee at face velocities of 100 . LFM at a $40 z$ sash opening. 


\section{CONCLUSIONS}

1. Based on the data contained in this report the Industrial Hygienists at ORNL, Y-12, and ORGDP are of the opinion that 100 LFM at a 408 sash opening is the minimum acceptable face velocity for laboratory hoods. Additionally certain operations will require greater face velocities. Note: This recommended face velocity (100 LFM), in no way contradicts the results of the Paducah study (KY-L-714), i.e., a hood designed to $50^{\circ}$ LFM with the sash full open would have a face velocity of at least 100 LFM with the sash at the 408 open point.

2. Professional judgement must be used in operations involving highly toxic chemicals. i.e., carrinngens, horyllium, oto.

3. Supportative data, i.e., breathing zone sampling, is neciessary on chemicals assigned a toxic index of 3 or 4 .

4. Because of the inherent problems of administering a hood classification system, the system should not be adopted formally but remain as Guidelines.

\section{GENERAL COMMENTS}

1. Laboratory hoods should be given a precise definition. For example: process ventilation in beryllium areas at $\mathrm{Y}-12$ have been incorrectly characterized as laboratory hoods. Process ventilation installed to prevent exposure to any chemical or physical stress must be carefully designed and their adequacy evaluated.

2. It should be reiterated that operations particularly at ORNL and Y-12 are many and varied. Programs tend to change rapidly. Ventilation requirements may also change just as rapidly depending on the process and/or the likelihood of exposure to a chemical or physical stress.

3. Informal discussions were held with industrial hygienists of LASL and ANL. The Engineering Section, Industrial Hygiene Group (H-5) at LASL advised that as a "general rule of thumb" 100 LFM is the minimum face velocity permitted for handing toxic chemicals and 125 LFM is the minimum face velocity for radioactive materials. 
They recognized that professional judgement was of considerable importance and was. used in relation to highly toxic chemicals such as beryllium or carcinogens. In these cases 125 to 150 LFM would be recommended. Breathing zone samples would be performed only if considered necessary by the staff. Industrial hygiene at ANL advised they also use "rule of thumb" of 100 LFM for most chemicals and 135 LFM for radioactive materials. They recognize that some chemicals are handled in certain operations which require greater flow rates. They also use a generous amount of professional judgement.

4. These conclusions and comments in no way contradict the riquirements of "General Design Criteria, Laboratory Buildings, FRDA Appendix 6301, Part II B (Approved November 20, 1972) which states: "An average air velocity of about $125 \mathrm{fpm}$ across the face of . each hood with the door opened to the full open operating position is recommended as a general rating. Higher or lower velocities may be justified under special circumstances depending upon the materials to be handled and their toxicity."

\section{ACKNOWLEDGEMENT}

The authors acknowledge the valuable comments and suggestions of J. T. Dufour, ERDA-ORO. 
TABLE 1

TEST HOOD DATA

\begin{tabular}{|c|c|c|c|c|c|}
\hline & $\begin{array}{c}\text { Hood } \\
\text { Face } \\
\text { Velocity } \\
\text { (LFM) }\end{array}$ & $\begin{array}{l}\text { Maximum } \\
\text { Breathing } \\
\text { Zone } \\
\text { (ppm) }\end{array}$ & $\begin{array}{l}\text { Average } \\
\text { Breathing } \\
\text { Zone } \\
\text { (ppm) }\end{array}$ & $\stackrel{8}{\mathrm{TLV}}$ & $\begin{array}{l}\text { TLV } \\
\text { (pFm) }\end{array}$ \\
\hline Carbon tetrachloride & & & & $\checkmark$ & \\
\hline 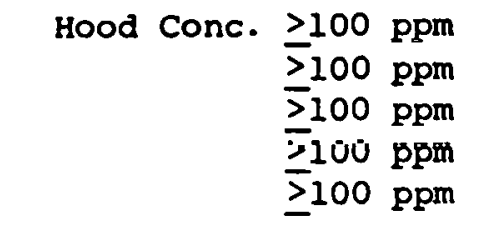 & $\begin{array}{r}150 \\
125 \\
100 \\
75 \\
50\end{array}$ & $\begin{array}{l}4.6 \\
4.6 \\
4.6 \\
60 \\
27\end{array}$ & $\begin{array}{c}4.6 \\
4.6 \\
4.6 \\
15.2 \\
9.5\end{array}$ & $\begin{array}{r}46 \\
46 \\
46 \\
152 \\
95\end{array}$ & 10 \\
\hline Perchloroethylene & & & & & \\
\hline $\begin{aligned} \text { Hood Conc. } & \geq 1000 \mathrm{ppm} \\
& \frac{\geq 1000 \mathrm{ppm}}{2} 500 \mathrm{ppm} \\
& \frac{21000 \mathrm{ppm}}{} \\
& \frac{2}{2} 500 \mathrm{ppm} \\
& \frac{21000 \mathrm{ppm}}{\geq 500 \mathrm{ppm}}\end{aligned}$ & $\begin{array}{r}150 \\
125 \\
100 \\
100 \\
75 \\
75 \\
50\end{array}$ & $\begin{array}{r}0.9 \\
0.9 \\
0.9 \\
1 \\
13 \\
110 \\
8\end{array}$ & $\begin{array}{r}0.9 \\
0.9 \\
0.9 \\
0.9 \\
3.4 \\
25.6 \\
2.2\end{array}$ & $\begin{array}{r}.9 \\
.9 \\
.9 \\
.9 \\
3.4 \\
25.6 \\
2.2\end{array}$ & 100 \\
\hline Freon 113 & & & & & \\
\hline $\begin{aligned} \text { Hood Conc. } & \geq 5,000 \mathrm{ppm} \\
& \geq 10,000 \mathrm{ppm} \\
& \geq 5,000 \mathrm{ppm} \\
& \geq 10,000 \mathrm{ppm} \\
& \frac{\geq}{\geq} 1,000 \mathrm{ppm} \\
& 2,000 \mathrm{ppm} \\
& \geq 1,000 \mathrm{ppm} \\
& \geq 1,000 \mathrm{ppm}\end{aligned}$ & $\begin{array}{r}150 \\
150 \\
125 \\
125 \\
100 \\
100 \\
75 \\
50\end{array}$ & $\begin{array}{l}0.7 \\
2 \\
0.7 \\
30 \\
15 \\
100 \\
55 \\
210\end{array}$ & $\begin{array}{l}0.7 \\
.86 \\
0.7 \\
4.4 \\
4.2 \\
17.9 \\
11.4 \\
43\end{array}$ & $\begin{array}{l}.07 \\
.09 \\
.07 \\
.44 \\
.42 \\
1.8 \\
1.1 \\
4.3\end{array}$ & 1000 \\
\hline
\end{tabular}

Note: These data are iliustrated graphically in Figures 1 and 2 . 


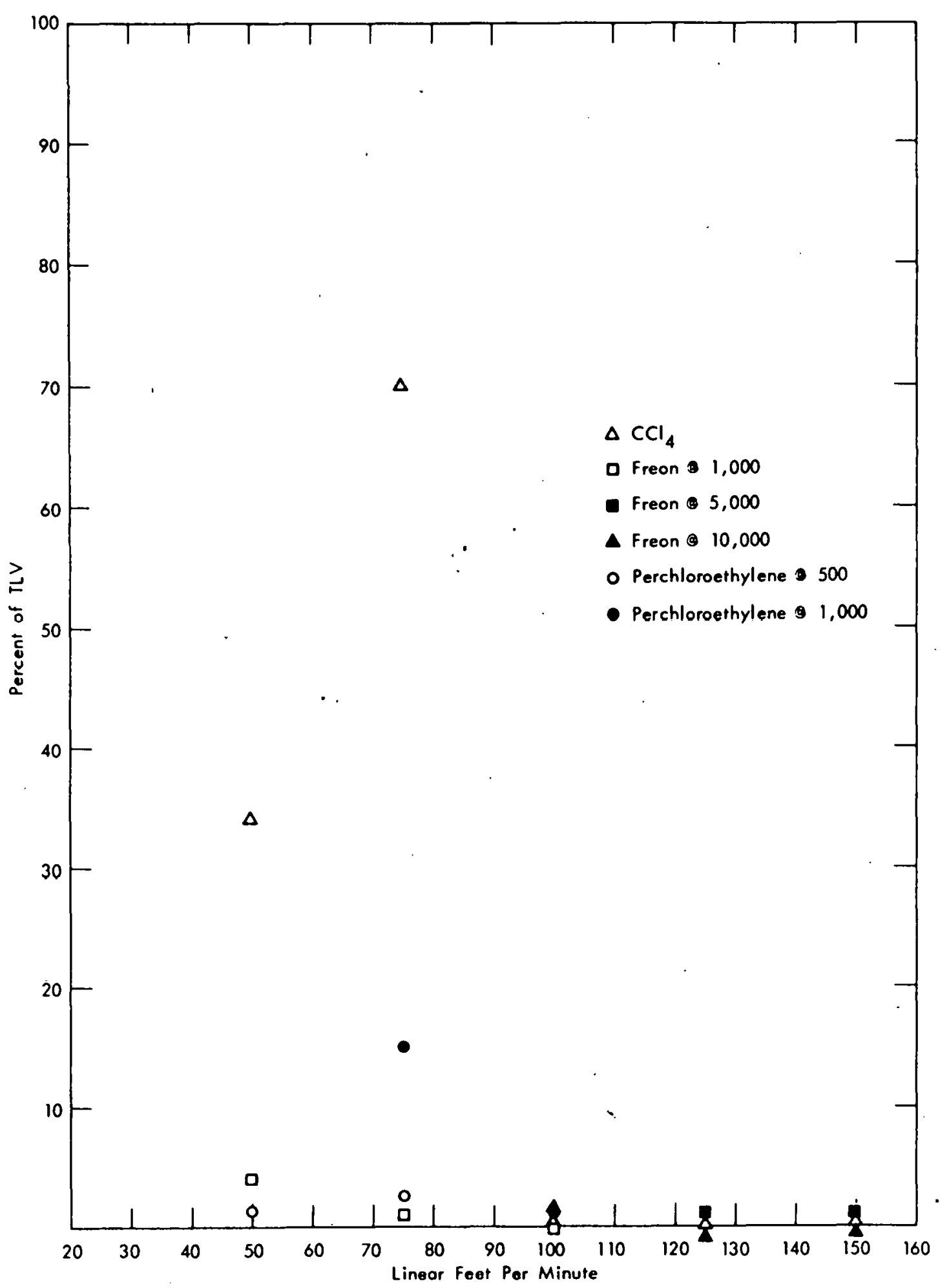

Figure 1. Summated Average Measured Concentrations. 


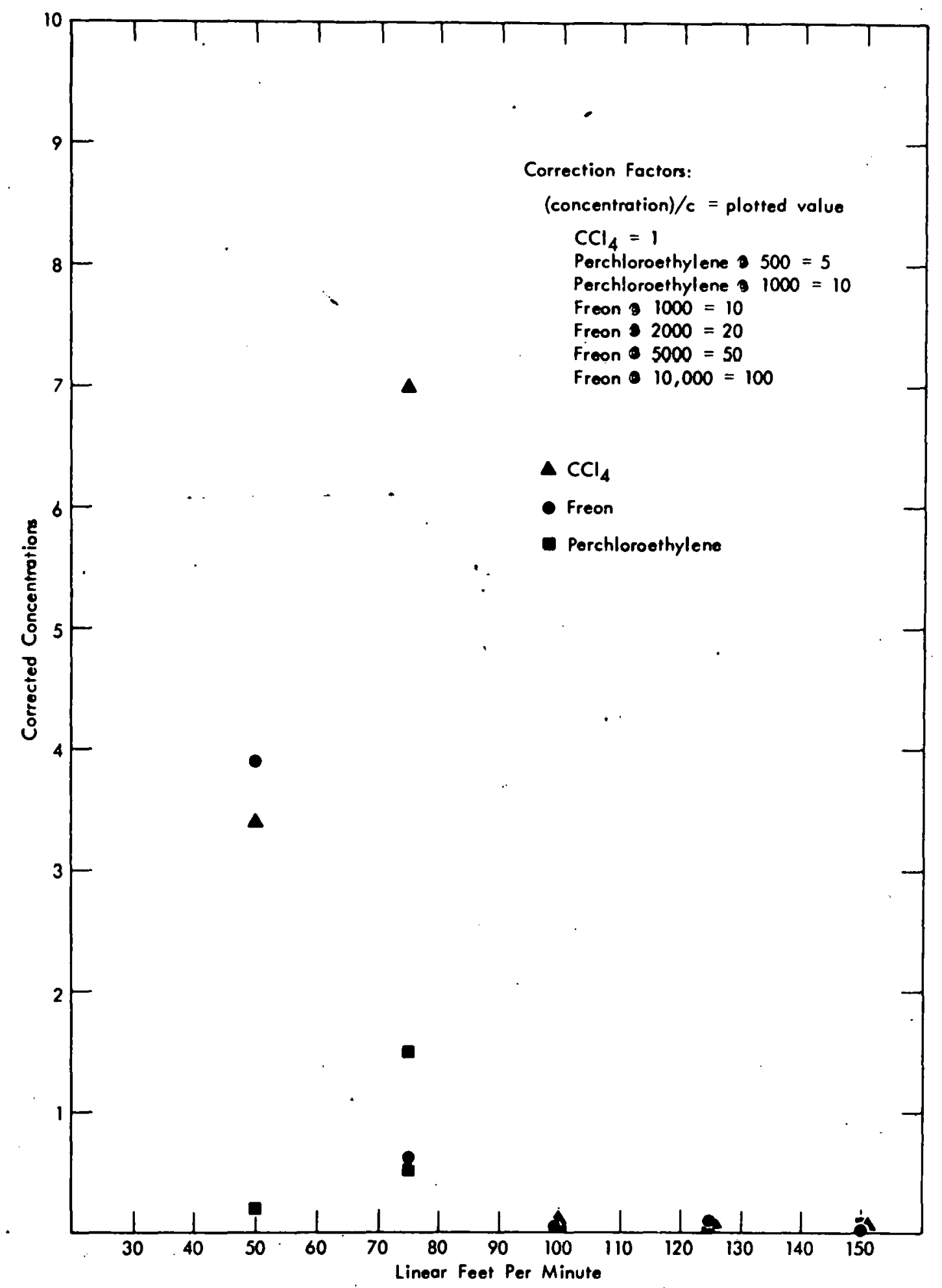

Figure 2. Summated Average Measured (Corrected) Concentrations. 
TABLE 2

AIR SAMPLING DATA FOR OPERATIONS CONDUCTED IN HOODS AT ORNL

\begin{tabular}{|c|c|c|c|c|c|}
\hline Date. & Location & Contaminant & Bz Conc. * & TLV & $\begin{array}{c}\text { Face } \\
\text { Velocity } \\
\text { (LFM) }\end{array}$ \\
\hline $2-21-73$ & $4508 \mathrm{Rm} \cdot 139 \mathrm{~A}$ & Benzene & $<1$ & 25 & 80 \\
\hline $2-21-73$ & $4508 \mathrm{Rm} \cdot 139 \mathrm{~A}$ & Benzene & 1 & 25 & 80 \\
\hline $2-21-73$ & $4508 \mathrm{Rm} \cdot 139 \mathrm{~A}$ & Benzene & 4.4 & 25 & 80 \\
\hline $2-16-73$ & $4508 \mathrm{~km} \cdot 140$ & Benzene & 2.8 & 25 & \\
\hline $4-30-73$ & 45005 & Dioxane & 1.4 & 100 & \\
\hline $4-30-73$ & $4500 s$ & Toluene & $<1$ & 100 & \\
\hline $5-9-73$ & 3502 Manipulator & Methyl Ethyl Ketone & 90 & 200 & 170 \\
\hline $5-9-73$ & 3502 Manipulator Shop & Methyl Ethyl Ketone & 40 & 200 & 170 \\
\hline $5-29-73$ & $4508 \mathrm{Rm} \cdot 139 \mathrm{~A}$ & Benzene & 7.5 & 25 & 110 \\
\hline $6-5-73$ & $4500 \mathrm{SR} \cdot \mathrm{C}-155$ & Toluene & $<1$ & 100 & 100 \\
\hline $6-14-73$ & 6010 Basement & xylene & $<1$ & 100 & 140 \\
\hline $11-28-73$ & 3502 Manipulator Shop & Methyl Ethyl Ketone & $<1$ & 200 & 130 \\
\hline $1-31-74$ & $4500 \mathrm{~S}$ Rm. E- 159 & Toluene & $<10$ & 100 & 90 \\
\hline $2-15-74$ & $4508 \mathrm{Rm} \cdot 139$ & Tetrabromoethane & $\leq .001$ & 1 & 85 \\
\hline $2-15-74$ & $4508 \mathrm{Rm} \cdot 139$ & Methylene Iodide & $\leq .05$ & 1 & 85 \\
\hline $2-19-74$ & $4500 \mathrm{Sm} \cdot \mathrm{F}-63$ & Toluene & $<10$ & 100 & 70 \\
\hline $2-19-74$ & $4508 \mathrm{Rm} \cdot 139 \mathrm{~A}$ & Benzene & $<2.5$ & 25 & 65 \\
\hline $2-21-74$ & 3502 Manipulator Shop & Methyl Ethyl Ketone & 30.3 & 200 & 75 \\
\hline $2-21-74$ & 3502 Manipulator Shop & Methyl Ethyl Ketone & $<20$ & 200 & 75 \\
\hline $2-25-74$ & 4500 S Rm. D- 263 & Tetrachloroethane & .31 & 5 & 140 \\
\hline $2-26-74$ & $4500 \mathrm{~N}$ Rm. E-17? & Carbon Tetrachloride & 1.3 & 10 & 140 \\
\hline $3-1-74$ & 4500N Rm. E-17 & Carbon Tetrachloride & $<1$ & 10 & 140 \\
\hline $3-1-74$ & $4500 \mathrm{~N} R \mathrm{~m} \cdot \mathrm{E}-17$ & Carbon Tetrachloride & 3.0 & 10 & 140 \\
\hline $4-3-74$ & $2001 \mathrm{Rm} .201$ & Hydrofluoric Acid & .5 & 3 & 110 \\
\hline $4-3-74$ & $2001 \mathrm{Rm} .201$ & Hydrofluoric Acid & $<.3$ & 3 & 110 \\
\hline $4-16-74$ & $4500 \mathrm{~N} \mathrm{Rm} \cdot \mathrm{A}-13$ & xylene & $\because<10$ & 100 & 130 \\
\hline $4-16-74$ & $4500 \mathrm{~N}$ Rm. A-13 & Dioxane & $<10$ & 100 & 130 \\
\hline $4-16-74$ & $\begin{array}{l}3502 \text { Manipulator Shop } \\
\text { - Breathing Zone Concer }\end{array}$ & $\begin{array}{l}\text { Methyl Ethyl Ketone } \\
\text { cration }\end{array}$ & $<20$ & 200 & 75 \\
\hline
\end{tabular}


TABLE 2 (Continued)

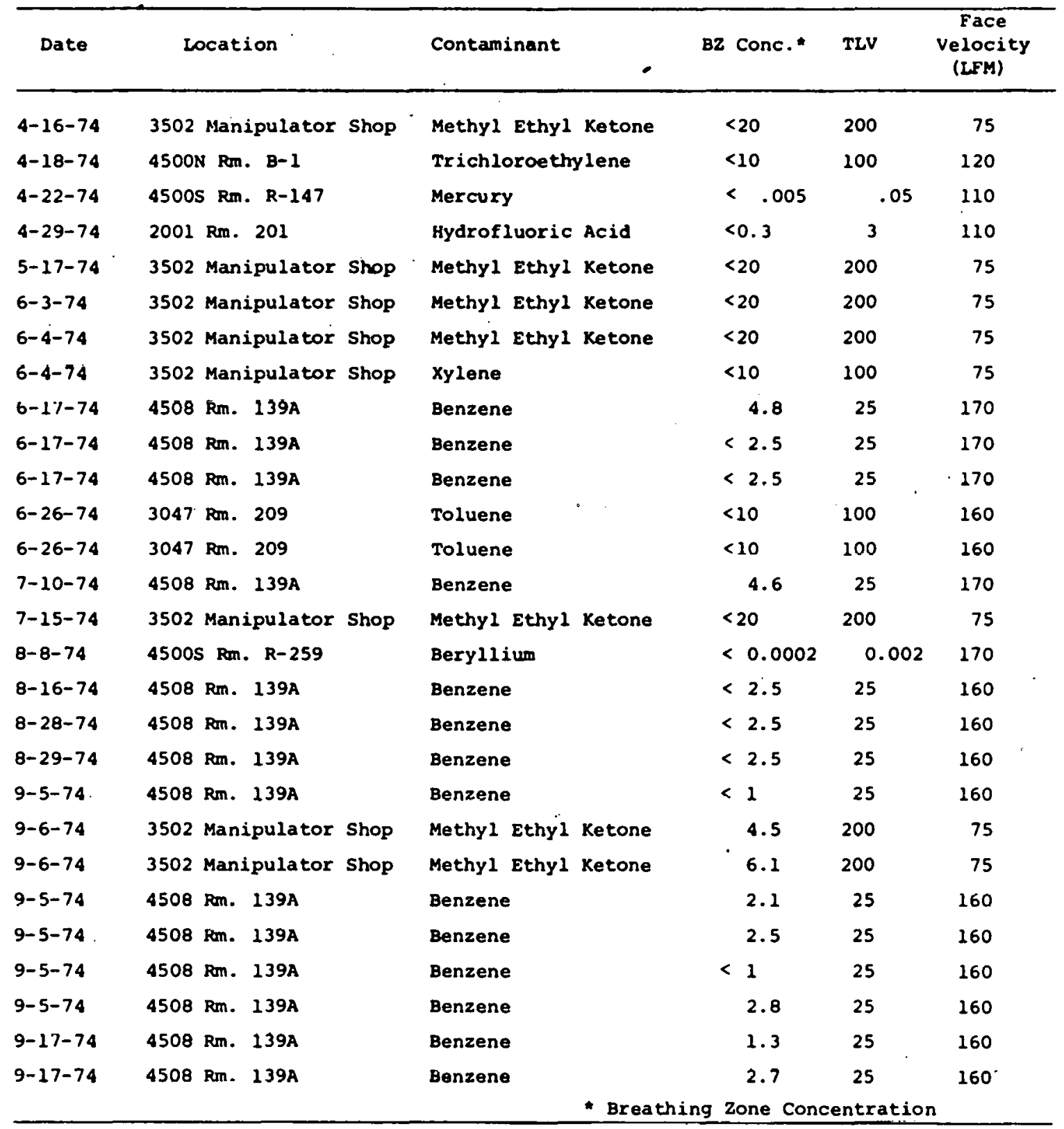


TABLE 3

AIR SAMPLING DATA FOR OPERATIONS CONDUCTED IN HOODS AT Y-12

\begin{tabular}{|c|c|c|c|c|c|}
\hline Date & Location & Contaminant & B2 Conc." & TLV & $\begin{array}{l}\text { Face } \\
\text { Velocity } \\
\text { (LFM) }\end{array}$ \\
\hline $\begin{array}{c}1-1-73 \text { to } \\
7-1-74\end{array}$ & $\begin{array}{l}9995 \\
\text { Hood \#63A }\end{array}$ & Beryllium. & $<0.1^{(1)}$ & $2 \mu g / m^{3}$ & 144 \\
\hline $\begin{array}{c}1-1-73 \text { to } \\
7-1-74\end{array}$ & $\begin{array}{l}9995 \\
\text { Hood \#63D }\end{array}$ & Beryllium & $<0.1^{(2)}$ & $2 \mu \mathrm{g} / \mathrm{m}^{3}$ & 186 \\
\hline $\begin{array}{l}1-1-73 \\
7-1-74\end{array}$ & $\begin{array}{l}9995 \\
\text { Hood \#64A }\end{array}$ & Beryllium & $<0.1^{(3)}$ & $2 \mu \mathrm{g} / \mathrm{m}^{3}$ & 100 \\
\hline $\begin{array}{c}1-1-73 \\
7-1-74\end{array}$ & $\begin{array}{l}9995 \\
\text { HoOd \#81A }\end{array}$ & Beryllium & $<0.1^{(4)}$ & $2 \mu \mathrm{g} / \mathrm{m}^{3}$ & 64 \\
\hline $\begin{array}{l}1-1-73 \\
8-1-74\end{array}$ & $\begin{array}{l}9995 \\
\text { Hood } \# 53 \mathrm{C} \text {. }\end{array}$ & Uranium & $<4 \mathrm{~d} / \mathrm{m} / \mathrm{m}^{3(5)}$ & $35 \mathrm{~d} / \mathrm{m} / \mathrm{m}^{3}$ & 74 \\
\hline $\begin{array}{l}1-1-73 \text { to } \\
8-1-74\end{array}$ & $\begin{array}{l}9995 \\
\text { Hood \#54A }\end{array}$ & Uranium & $<4 \mathrm{~d} / \mathrm{m} / \mathrm{m}^{3(6)}$ & $35 \mathrm{~d} / \mathrm{m} / \mathrm{m}^{3}$ & 71 \\
\hline
\end{tabular}

(1) Represents results of 56 samples."

(2) Represents results of 57 samples.

(3) Represents results of 55 samples.

(4) Represents results of 53 samples.

(5) Represents results of 190 samples.

(6) Kepresents results of 191 samples.

* Breathing zone concentration 
TABLE 4

AIR SAMPLING DATA FOR OPERATIONS CONDUCTED IN HOODS AT Y-12

\begin{tabular}{|c|c|c|c|c|c|}
\hline Date & Location & Contaminant & Bz Conc." & TLV & $\begin{array}{l}\text { Face } \\
\text { Velocity } \\
\text { (LFM) }\end{array}$ \\
\hline $10-23-70$ & $\begin{array}{l}9201-5,3 \text { rd Floor } \\
\text { Hood } \# 61\end{array}$ & Beryllium & $9.3 \mu \mathrm{g} / \mathrm{m}^{3}$ & $2 \mu \mathrm{g} / \mathrm{m}^{3}$ & 134 \\
\hline $10-26-70$ & $\begin{array}{l}9201-5, \text { 3rd Floor } \\
\text { Hood } \# 61\end{array}$ & Beryllium & $3.3 \mu \mathrm{g} / \mathrm{m}^{3}$ & $2 \mu g / \mathrm{m}^{3}$ & 134 \\
\hline $11-1-70$ & $\begin{array}{l}9201-5,3 \text { rd Floor } \\
\text { Hood \#61 }\end{array}$ & Beryllium & $20.6 \mu \mathrm{gg} / \mathrm{m}^{3}$ & 2. $\mu \mathrm{g} / \mathrm{m}^{3}$ & 134 \\
\hline $11-4-70$ & $\begin{array}{l}9201-5,3 \text { rd Floor } \\
\text { Hood } \# 61\end{array}$ & Beryllium & $2.9 \mu \mathrm{g} / \mathrm{m}^{3}$ & $2 \mu \mathrm{g} / \mathrm{m}^{3}$ & 134 \\
\hline $11-6-70$ & $\begin{array}{l}\text { y201-5, 3rd Floor } \\
\text { Hood } \# 61\end{array}$ & Beryllium & $2.2 \mu \mathrm{g} / \mathrm{m}^{3}$ & $2 \mu g / \mathrm{m}^{3}$ & 134 \\
\hline $11-9-70$ & $\begin{array}{l}9201-5,3 \text { rd Floor } \\
\text { Hood \#61 }\end{array}$ & Beryllium & $5.2 \mu \mathrm{g} / \mathrm{m}^{3}$ & $2 \mu g / m^{3}$ & 134 \\
\hline $11-10-70$ & $\begin{array}{l}9201-5,3 \text { rd Floor } \\
\text { Hood } \# 61\end{array}$ & Beryllium & $8.8 \mu \mathrm{g} / \mathrm{m}^{3}$ & $2 \mu \mathrm{g} / \mathrm{m}^{3}$ & 134 \\
\hline $11-11-70$ & $\begin{array}{l}9201-5,3 r d \text { Floor } \\
\text { Hood } \# 61\end{array}$ & Beryllium & $2.0 \mu \mathrm{g} / \mathrm{m}^{3}$ & $2 \mu g / m^{3}$ & 134 \\
\hline $11-13-70$ & $\begin{array}{l}9201-5, \text { 3rd Floor } \\
\text { hood } \# 61\end{array}$ & Beryllium & $6.4 \mu \mathrm{g} / \mathrm{m}^{3}$ & $2 \mu \mathrm{g} / \mathrm{m}^{3}$ & 134 \\
\hline $2-1-70$ & $\begin{array}{l}9201-5,3 \text { rd Floor } \\
\text { Hood \#61 }\end{array}$ & Beryllium & $0.1 \mu \mathrm{g} / \mathrm{m}^{3}$ & $2 \mu g / m^{3}$ & 134 \\
\hline $10-22-70$ & 9201-5, Hood \#62 & Beryllium & $0.1 \mu \mathrm{g} / \mathrm{m}^{3}$ & $2 \mu \mathrm{g} / \mathrm{m}^{3}$ & 124 \\
\hline $10-23-70$ & $9201-5$, Hood $\# 62$ & Beryllium & $1.7 \mu \mathrm{g} / \mathrm{m}^{3}$ & $2 \mathrm{\mu g} / \mathrm{m}^{3}$ & 124 \\
\hline $10-26-70$ & 9201-5, Hood \#62 & Beryllium & $3.3 \mu \mathrm{g} / \mathrm{m}^{3}$ & $2 \mu \mathrm{g} / \mathrm{m}^{3}$ & 124 \\
\hline $11-1-70$ & $9201-5$, Hood $\$ 62$ & Beryllium & $2.0 \mu \mathrm{g} / \mathrm{m}^{3}$ & $2 \mu g / \mathrm{m}^{3}$ & 124 \\
\hline $11-4-70$ & $9201-5$, Hood $\# 62$ & Beryllium & $2.0 \mu \mathrm{g} / \mathrm{m}^{3}$ & $2 \mu \mathrm{g} / \mathrm{m}^{3}$ & 124 \\
\hline $11-6-70$ & $9201-5$, Hood \#62 & Beryllium & $0.5 \mathrm{jg} / \mathrm{m}^{3}$ & $2 \mu \mathrm{g} / \mathrm{m}^{3}$ & 124 \\
\hline $11-9-70$ & $9201-5$, Hood \#62 & Beryllium & $2.2 \mu \mathrm{g} / \mathrm{m}^{3}$ & $2 \mu \mathrm{g} / \mathrm{m}^{3}$ & 124 \\
\hline $11-11-70$ & $9201-5$, Hood $\# 62$ & Beryllium & $16.7 \mu \mathrm{g} / \mathrm{m}^{3}$ & $2 \mu \mathrm{g} / \mathrm{m}^{3}$ & 124 \\
\hline $11-10-70$ & 9201-5, Hood \#62 & Beryllium & $8.7 \mu \mathrm{g} / \mathrm{m}^{3}$ & $2 \mu g / m^{3}$ & 124 \\
\hline $11-13-70$ & 9201-5, Hood \#62 & Beryllium & $0.4 \mu \mathrm{g} / \mathrm{m}^{3}$ & $2 \mu g / \mathrm{m}^{3}$ & 124 \\
\hline $8-1-73$ & $\begin{array}{l}9201-5, \text { 2nd Floor } \\
\text { Hood } \| 22\end{array}$ & Beryllium & $0.68 \mu \mathrm{gg} / \mathrm{m}^{3}$ & $2 \mu \mathrm{g} / \mathrm{m}^{3}$ & 124 \\
\hline $8-1-73$ & $\begin{array}{l}9201-5, \text { 2nd Flcor } \\
\text { HoOd } \# 20\end{array}$ & Beryllium & $0.28 \mu \mathrm{y} / \mathrm{m}^{3}$ & $2 \mu \mathrm{g} / \mathrm{m}^{3}$ & 124 \\
\hline
\end{tabular}


TABLE 4 (Continued)

\begin{tabular}{llllll}
\hline Date & Location & Contaminant & Bz Conc. & TLV & $\begin{array}{c}\text { Face } \\
\text { Velocity } \\
\text { (LFM) }\end{array}$ \\
\hline $8-1-73$ & $\begin{array}{l}9201-5, \text { 2nd Floor } \\
\text { Hood \#20 }\end{array}$ & Beryllium & $0.66 \mu \mathrm{g} / \mathrm{m}^{3}$ & $2 \mu \mathrm{g} / \mathrm{m}^{3}$ & 124 \\
$8-2-73$ & $\begin{array}{l}9201-5,2 \text { 2nd Floor } \\
\text { Hood \#22 }\end{array}$ & Beryllium & $0.47 \mu \mathrm{g} / \mathrm{m}^{3}$ & $2 \mu \mathrm{g} / \mathrm{m}^{3}$ & 124 \\
$8-2-73$ & $\begin{array}{l}9201-5,2 \text { 2nd Floor } \\
\text { Hood \#19 }\end{array}$ & Beryllium & $0.33 \mu \mathrm{g} / \mathrm{m}^{3}$ & $2 \mu \mathrm{g} / \mathrm{m}^{3}$ & 124 \\
$8-2-73$ & $\begin{array}{l}9201-5,2 \text { 2nd Floor } \\
\text { Hood \#19 }\end{array}$ & Beryllium & $0.31 \mu \mathrm{g} / \mathrm{m}^{3}$ & $2 \mu \mathrm{g} / \mathrm{m}^{3}$ & 124 \\
\hline
\end{tabular}

- Breathing Zone Concentration 
TABLE 5

AIR SAMPLING DATA FOR OPERATIONS CONDUCTED IN HOODS AT K-25

\begin{tabular}{|c|c|c|c|c|c|}
\hline Date & Location & Contaminant & B2 Conc." & TLV & $\begin{array}{c}\text { Face } \\
\text { Velocity } \\
\text { (LFM) }\end{array}$ \\
\hline $10-1-74$ & $K-1004-A-19-248069^{\circ}$ & Trichloroethane & $<50$ ppm & 350 & 70 \\
\hline $10-1-74$ & $K-1004-A-18-259019$ & Nitrous Fumes & 0 ppm & 5 & 85 \\
\hline $10-1-74$ & $K-1004-A-19-259020$ & Ammonium Hydroxide & 0 ppm & 25 & 85 \\
\hline $10-1-74$ & $K-1004-A-19-259021$ & Hydrogen Chloride & $0 \mathrm{ppm}$ & 5 & 110 \\
\hline $10-8-74$ & $K-1004-L-23-248533$ & Arsetones & $<100 \mathrm{ppm}$ & 1000 & Su \\
\hline $10-8-74$ & $K-1004-L-9-248535$ & Acetone & $<100 \mathrm{ppm}$ & 1000 & 95 \\
\hline $10-8-74$ & $K-1004-L-9-248534$ & Acetone & $<100 \mathrm{ppm}$ & 1000 & 110 \\
\hline $10-8-74$ & $K-1004-L-6-24857$ & Acetone & $<100 \mathrm{ppm}$ & 1000 & 80 \\
\hline $10-8-74$ & $K-1006-B-102-249674$ & Benzenc & o ppm & 10 & 40 \\
\hline $10-0-74$ & $k=100 b-c-101-249 / 26$ & Hydrogen Chloride & 0 ppm & 5 & 90 \\
\hline $10-8-74$ & $k-1006-c-108-249688$ & Acetone & $<100 \mathrm{ppm}$ & 1000 & 125 \\
\hline $10-8-74$ & $x-1006-c-104-249723$ & Hydrogen Floride & 0 ppm & 3 & 85 \\
\hline $10-8-74$ & $k-1006-c-108-249715$ & Hydrngen Chloride & $0 \mathrm{ppm}$ & 5 & 90 \\
\hline $10-31-74$ & $K-1004-D-17-187105$ & Trichloroethane & $<50 \mathrm{ppm}$ & 350 & 50 \\
\hline $10-31-74$ & $K-1004-D-31-187195$ & Hydrogen Floride & $0 \mathrm{ppm}$ & 3 & 110 \\
\hline $10-2-74$ & $K-1004-B-150-248092$ & Acetone & $<100 \mathrm{ppm}$ & 1000 & 125 \\
\hline $10-1-74$ & K-1004-B-108-255561 & Hydrogen Chloride & $0 \mathrm{ppm}$ & 5 & 95 \\
\hline $10-1-74$ & $K-1004-B-108-255562$ & Hydrogen Chloride & 0 ppm & 5 & 120 \\
\hline $10-1-74$ & $K-1004-B-108-255559$ & Hydrogen Chloride & 0 ppm & 5 & 65 \\
\hline $10-1-74$ & $K-1004-8-108-255560$ & Hydrogen Chloride & 0 ppm & 5 & 100 \\
\hline $9-27-74$ & $K-1004-B-118-255573$ & Hydrogen Chloride & $0 \mathrm{ppm}$ & 5 & 85 \\
\hline $9-27-74$ & $K-1004-B-114-248008$ & Hydrogen Floride & 0 ppm & 3 & 50 \\
\hline $9-27-74$ & $K-1004-B-103-248018$ & Hydrogen Floride & $0 \mathrm{ppm}$ & 3 & 65 \\
\hline $9-27-74$ & $K-1004-B-103-246780$ & Hydrogen Chloride & 0 ppm & 5 & 205 \\
\hline $10-31-74$ & $K-1004-D-28-248088$ & Hydrogen Chloride & 0 ppm & 5 & 40 \\
\hline $10-3-74$ & $K-1101-L a b-248547$ & Hydrogen Chloride & $0 \mathrm{ppm}$ & 5 & 75 \\
\hline $10-3-\% 4$ & $K-1.413-L a b-258400$ & Hydrogen Cinloride & $0 \mathrm{ppm}$ & 5 & 65 \\
\hline $10-3-74$ & $K-1420-L a b-258525$ & Hydrogen Chloride & $0 \mathrm{ppm}$ & 5 & 40 \\
\hline $10-3-74$ & $K-1010-A-253390$ & Perchloroethylene & 0 ppm & 100 & 30 \\
\hline $10-3-74$ & $K-1231-104$ & Hydrogen Chloride & $0 \mathrm{ppm}$ & 5 & 75 \\
\hline $9-30-74$ & $\mathrm{~K}-1004-\mathrm{C}-210-258858$ & Trichloroethane & $<100 \mathrm{ppm}$ & 350 & 60 \\
\hline $9-30-74$ & $k-1004-C-212-258857$ & Hydrogen Floride & 0 ppm & 3 & 55 \\
\hline $9-30-74$ & $K-1004-C-219-256053$ & Hydrogen Floride & $0 \mathrm{ppm}$ & 5 & 85 \\
\hline $9-30-74$ & $k-1004-c-220-256051$ & Hydrogen Chloride & 0 ppm & 5 & 100 \\
\hline $9-30-74$ & $k-1004-C-215-255237$ & Hydrogen Floride & 0 ppm & 3 & 120. \\
\hline $9-30-74$ & K-1004-C-215-255237 & Hydrogen Chloride & 0 ppm & 5 & 120 \\
\hline $9-30-74$ & $K-1004-C-215-255237$ & Nitrous Fumes & $0 \mathrm{ppm}$ & 5 & 120 \\
\hline $9-30-74$ & $k-1004-c-215-255237$ & Acetone & $<100$ ppm & 1000 & 120 \\
\hline
\end{tabular}


APPENDIX A

R. G. Jordan

$\operatorname{March} 25,1974$

UCND - Safety and Envionmental Protection

$9704-2, \quad Y-12$

R. G. Affel

F. R. Bruce

LABORATORY HOODS

T. A. Lincoln

CLASSIFICATION GUIDELINES

On December 4, 1973, J. F. Morehead $(Y-12)$, H. F. Higdon (ORGDP) and I met to discuss problems of hood classifications. Although Turok (PGDP) was not in attendance he has reviewed the "Classification Guidelines" and is in agreement with us. We suggest that the attached Guidelines be used when or if we are required to classify hoods in terms of the toxic materials being handled. This system will, we believe, result in the least confusion. As experienced is gained we may need to make changes in the TLV ranges for each class.

I would hope that these guidelines would not end "being carved in stone." For example, if it could be demonstrated under actual use conditions that the TLV were not exceeded, a class II hood could be ised for any given material.

N. E. Bolton

ORNL, Industrial Hygiene Department

NEB : rCW

Attachments 
LABORATORY HOODS

\section{Classification Guidelines}

\section{Selection Requirements}

For purposes of selection of the class hood required the following definitions shall be used:

\section{Toxicity}

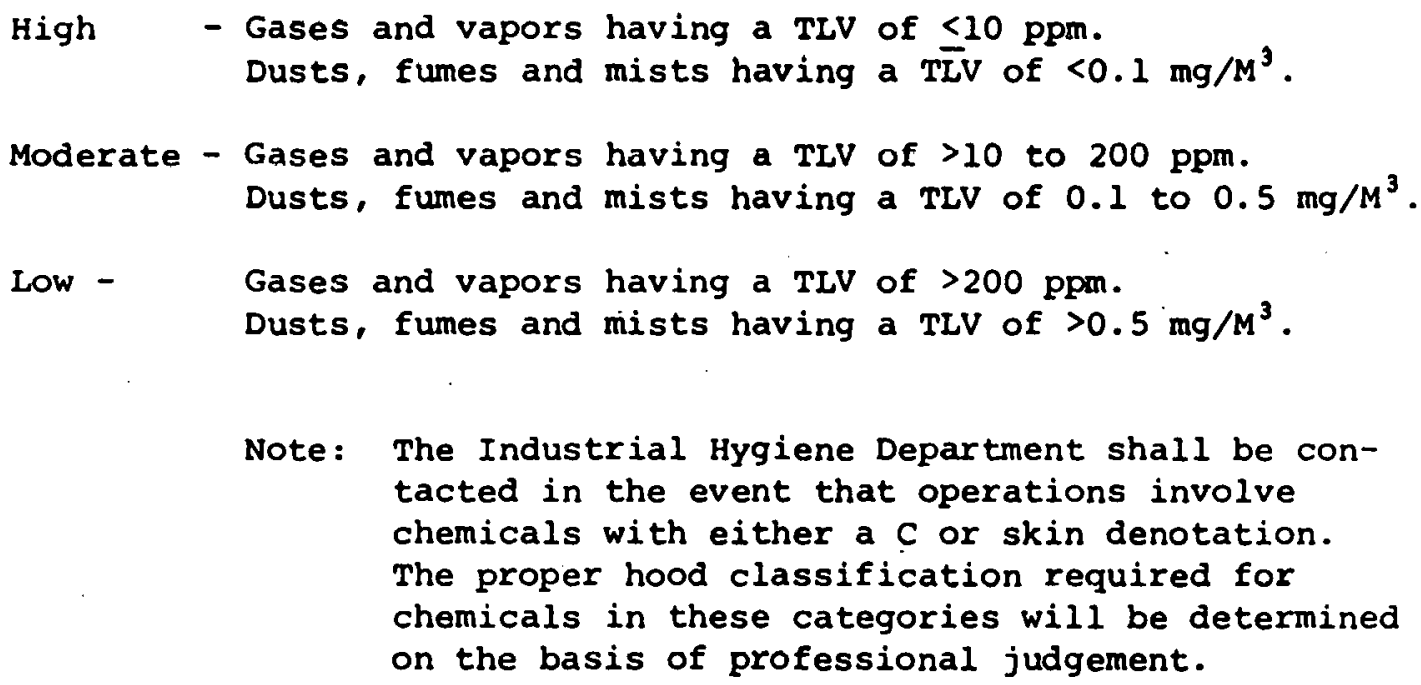


IABGRATORY HOODS

Classification Guidelines

Page 2

Alternate Selection Requirements

If TLV's are not established, the following data may be used to determine hood classification requirements.

\begin{tabular}{|c|c|c|c|c|c|}
\hline $\begin{array}{l}\text { Hood Classification } \\
\text { Requirements }\end{array}$ & $\begin{array}{l}\text { Toxic } \\
\text { Index }\end{array}$ & $\begin{array}{l}\text { LDso Single Oral } \\
\text { Dose Rats } \\
\text { mg/Kg }\end{array}$ & $\begin{array}{l}\text { ICso } 4 \mathrm{hr} \text {. } \\
\text { Inhalation } \\
\text { ppm }\end{array}$ & $\begin{array}{l}\mathrm{LD}_{50} \mathrm{Skin} \\
\mathrm{mg} / \mathrm{Kg}\end{array}$ & $\begin{array}{l}\text { Approximate } \\
\text { TLV Range }\end{array}$ \\
\hline \multirow[t]{2}{*}{ I } & 1 & $501-15,000$ & $1,001-100,000$ & $350-22,600$ & $\begin{array}{l}>200 \mathrm{ppm} \text { or } \\
>0.5 \mathrm{mg} / \mathrm{M}^{3}\end{array}$ \\
\hline & 2 & $50.1-500$ & $101-1,000$ & $44-349$ & \\
\hline II & 3 & $1.1-50$ & $11-100$ & $5.1-43$ & $\begin{array}{l}\geq 10-<200 \mathrm{ppm} \text { or } \\
\geq 0.1-<0.5 \mathrm{mg} / \mathrm{m}^{3}\end{array}$ \\
\hline III & 4 & $\leq 1$ & $\leq 10$ & $\leq 5$ & $\begin{array}{l}<10 \mathrm{ppm} \text { or } \\
<0.1 \mathrm{mg} / \mathrm{M}^{3}\end{array}$ \\
\hline
\end{tabular}


LABORATORY HOODS

Classification Guidelines

Page 3

\section{Classification}

Hoods for general laboratory usage shall be classified in the following categories:

$$
\begin{aligned}
& \text { Class } 1 \text { - For handling a) non-toxic or low toxicity materials } \\
& \text { or b) higher toxic materials in sealed containers } \\
& \text { and/or closed systems. }
\end{aligned}
$$

\section{Airflow Requirements}

Laboratory hoods, when operating with the sash set at the Normal Operating Opening shall have face velocities as follows:

$$
\begin{aligned}
\text { Class } 1 \text { - } & \text { Design airflow shall be } 60 \mathrm{ft} / \mathrm{min} . \\
& \text { Minimum airflow shall be } 50 \mathrm{ft} / \mathrm{min} . \\
\text { Class } 2 \text { - } & \begin{array}{l}
\text { Design airflow shall be } 100 \mathrm{ft} / \mathrm{min} . \\
\text { Minimum airflow shall be } 80 \mathrm{ft} / \mathrm{min} .
\end{array} \\
\text { Class } 3 \text { - } & \begin{array}{l}
\text { Design airflow shall be } 150 \mathrm{ft} / \mathrm{min} . \\
\text { Minimum airflow shall be } 125 \mathrm{ft} / \mathrm{min} .
\end{array}
\end{aligned}
$$


LABORATORY HOODS

Classifisation Guidelines

page 4

Hood Identification

All Class I Hoods shall bear the following label:

CLASS I

Air flow requirements: Minimum $50 \mathrm{ft} / \mathrm{min}$., design 60

$\mathrm{ft} / \mathrm{min}$. for chemicals in the following category:

Gases and vapors having a TLV of $\geq 200 \mathrm{ppm}$.

Dusts, fumes, and mists having a ' $1 \mathrm{~L} L \mathrm{l}$ of $>0.5 \mathrm{mg} / \mathrm{M}^{3}$.

For information: Call Industrial Hygiene Department.

All Class II Hoods shall bear the following label:

CIASS I I

Air flow requirements: Minimum $80 \mathrm{ft} / \mathrm{min} .$, design 100

ftimin. for chemicals in the following category:

Gases and vapors having a TLV of $\geq 10$ to $<200$ ppm

Dusts, fumes, and mists having a TLV of $>0.1$ to $<0.5 \mathrm{mg} / \mathrm{M}^{3}$.

For information: Call Industrial Hygiene Department.

All Class III Hoods shall bear the following label:

CENSS III

Air flow requirements: Minimum $125 \mathrm{ft} . / \mathrm{min} .$, desigr

$150 \mathrm{ft} / 11 \mathrm{il}$, for chemicals in the following category:

Gases and vapors having a TLV of $<10$ ppm.

Dusts, fumes, and mists having a TLV of $<0.1 \mathrm{mg} / \mathrm{M}^{3}$.

For informaticn: Call Industrial Hygiène Department. 


\section{THIS PAGE}

\section{WAS INTENTIONALLY \\ LEFT BLANK}


APPENDIX $\mathrm{B}$

DESCRIPTION OF THE Y-12 LABORATORY TEST HOOD 
DESCRIPTION OF THE Y-12 LABORATORY TEST HOOD

I. TESTING SET-UP

The $\mathrm{Y}-12$ Test Hood (NII, Model H-804) is located in the northwest corner of Room 43, Building 9766. A counterbalanced, vertical sash is installed on the hood which can be easily moved up and down to vary the hood opening or close it off entirely. In order to simulate the actual conditions in hoods throughout the plant, several items were kept within the hood during testing. In the back right corner was a $14 \mathrm{oz}$. can of cleanser, a 1 liter flask, and the Scott-Davis Halide Meter (11" $x$ 7" $x$ 7"). In the back left corner was a 1 quart bottle, a 2 quart bottle, and a 1 gallon metal safe can. These are mentioned because any item within a hood in some way affects the airflow. A picture of the hood model with diagrams of unrestricted airflow is shown in Figure 1.

II. TEST GASES

Three materials were chosen to be tested:

Carbon Tetrachloride $\left(\mathrm{CCL}_{4}\right)$

TLV - $10 \mathrm{ppm}$ or $65 \mathrm{mg} / \mathrm{m}^{3}$

Boiling Point $-170^{\circ} \mathrm{F}$.

Vapor Density (air $=1$ ) -5.32

Lower Detection Limit $=4.6 \mathrm{ppm}$ or $30 \mathrm{mg} / \mathrm{m}^{3}$

Perchlornethylene $\left(\mathrm{C}_{2} \mathrm{Cl}_{4}\right)$

TLV - $100 \mathrm{ppm} / 670 \mathrm{mg} / \mathrm{m}^{3}$

Boiling Point $-248^{\circ} \mathrm{F}$.

Vapor Density, (air = 1) - 5.67

Lower Detection Limit $=0.9 \mathrm{ppm}$ or $6.03 \mathrm{mg} / \mathrm{m}^{3}$

Freon 113, Trichlorotrifluorethane $\left(\mathrm{CCl}_{2} \mathrm{~F}-\mathrm{CClF}{ }_{2}\right)$

TLV - $1,000 \mathrm{ppm} / 7,600 \mathrm{mg} / \mathrm{m}^{3}$

Boiling Point $-117^{\circ} \mathrm{F}$.

Vapor Density (air = 1) - 0.46

Lower Detection Limit $=0.7 \mathrm{ppm}$ or $5.32 \mathrm{mg} / \mathrm{m}^{3}$

These gases were picked primarily because of the range of TLV's represented, and the availability of instrumentation. 


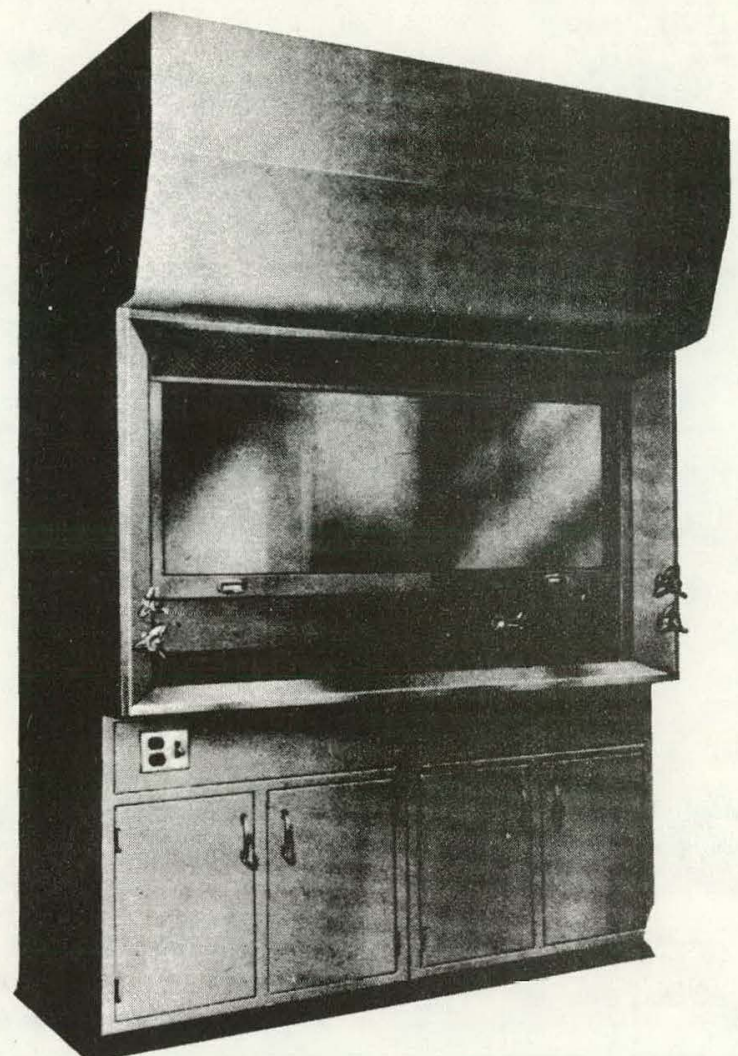

H-804 V.I.P. HOOD

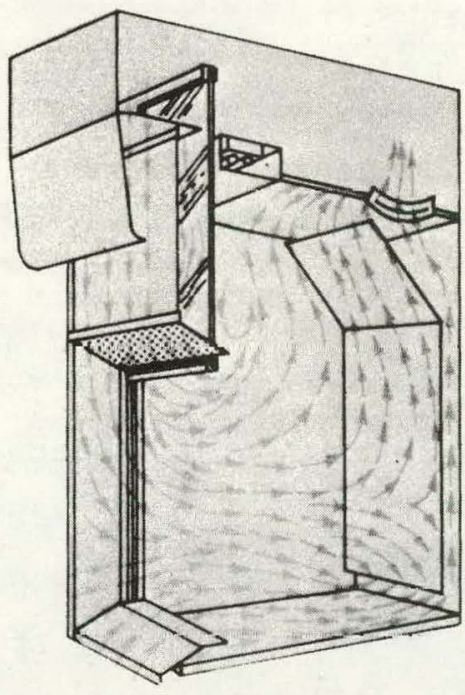

SASH IN OPEN POSITION

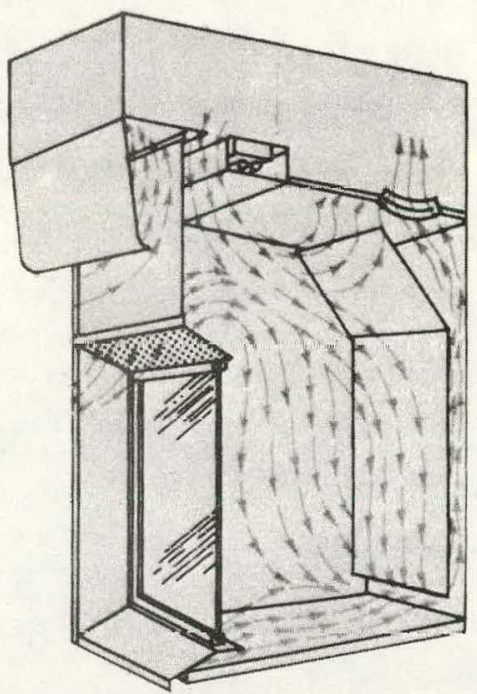

SASH IN CLOSED POSITION

Figure 1. Fume Hood-V. I.P. Series H-800.

Note: Specific Design Data and Operating Characteristics of this Fume Hood are given on page 66 of Catalog of Laboratory Furniture, Inc. (Copyright 1973). 


\section{INSTRUMENT CALIBRATION}

The Scott-Davis Halide Meter, used to test concentrations inside the hood and the Miran Infrared Gas Analyzer, used to test breathing zone concentrations at the face of the hood were both calibrated at the Y-12 Plant Laboratory (Building 9995). Calibration curves were provided for both instruments for the three test gases. The Alnor Thermo-anemometer was calibrated by $\mathrm{Y}-12$ electrical maintenance and a curve provided. The technique used is given in Attachment 1 . Later, another Alnor Thermo-anemometer was used which read in actual feet per minute making a curve unnecessary.

\section{TESTING PROCEDURE}

The face velocity of the test hood was adjusted by means of a damper to approximately the desired measurement. Measurements were normally taken with the vertical sash at its maximum height (face opening= $292 / 3 " x 61 ")$. The plane of the hood face was divided into 8 equally spaced sections. A velocity reading was taken in each section with an Alnor Thermo-anemometer and the results averaged as indicated in Table 1. Table 2 illustrates the effect of the sash position on face velocity. This effect is graphically displayed in Figure 2.

Once a given face velocity was determined, a 3-minute smoke bomb was placed in the hood for a visual check of leakage before any testing was done. If a significant amount of smoke escaped from the hood opening, no testing was performed at that face velocity. For example: at 25 fpm face velocity large amounts of smoke escaped from the hood; therefore, no testing was done at this velocity. A smoke test rating of "good" was given to those face velocities where no visible smoke escaped from the hood opening.

After a face velocity was determined and found reasonably safe, an amount of the material to be tested was placed in a stainless steel electric fry pan, used to generate the tested vaports. All testing was done with the fry pan in 2 positions -- in the middle of the hood and in the extreme left of the hood-- both positions were 4 inches from the lower edge of the hood face. At the extreme right inside the hood was a sink which precluded testing in that position. 
TABLE 1

THERMO-ANEMOMETER READINGS FOR TESTED FACE VELOCITIES

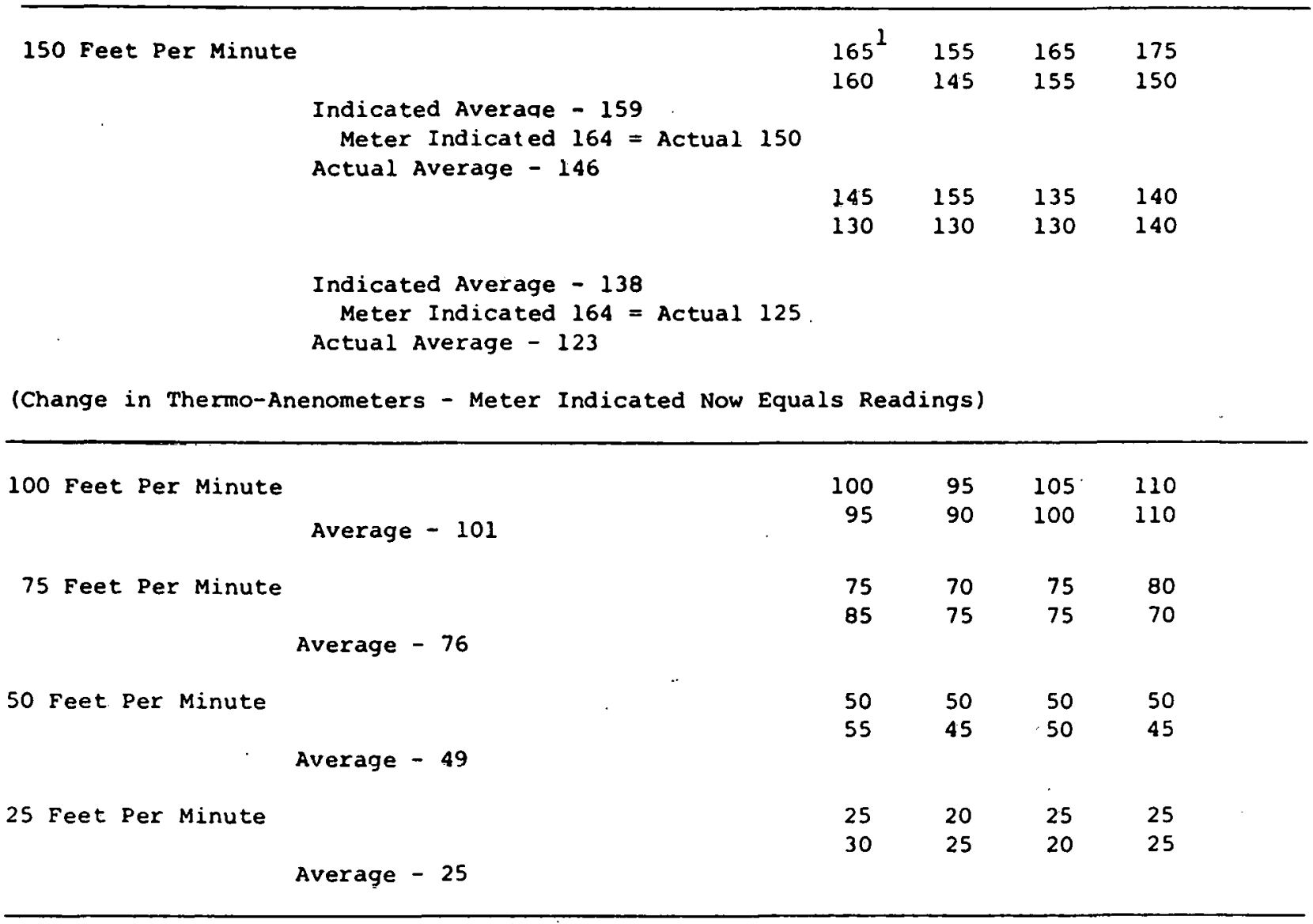

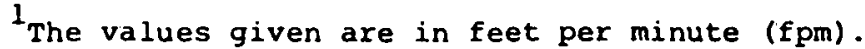


TABLE 2

THERMO-ANEMOMETER READINGS FOR CHANGES IN HEIGHT OF VERTICAL SASH

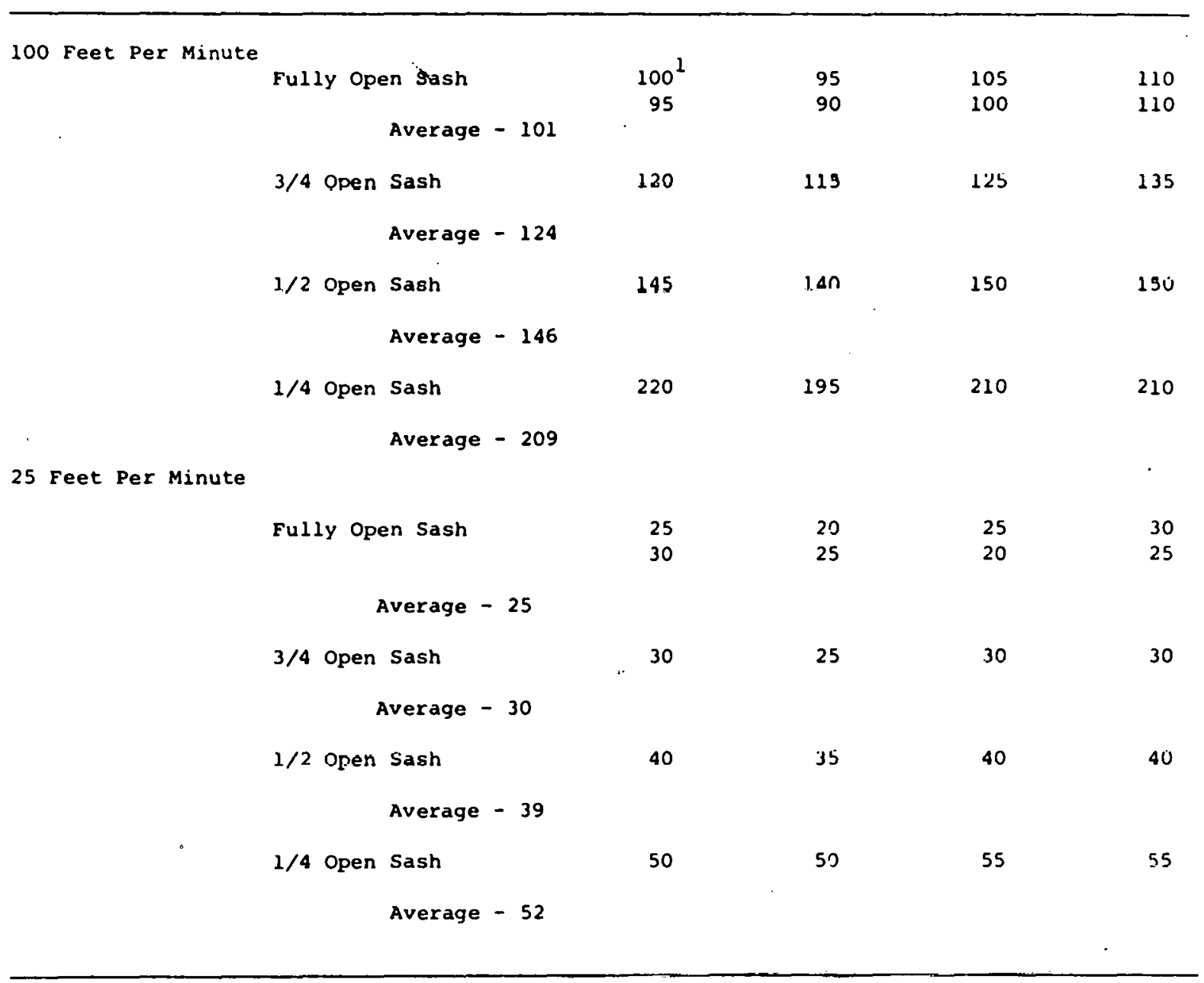

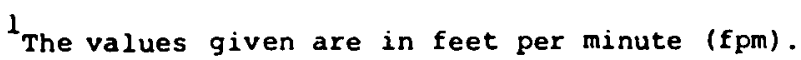




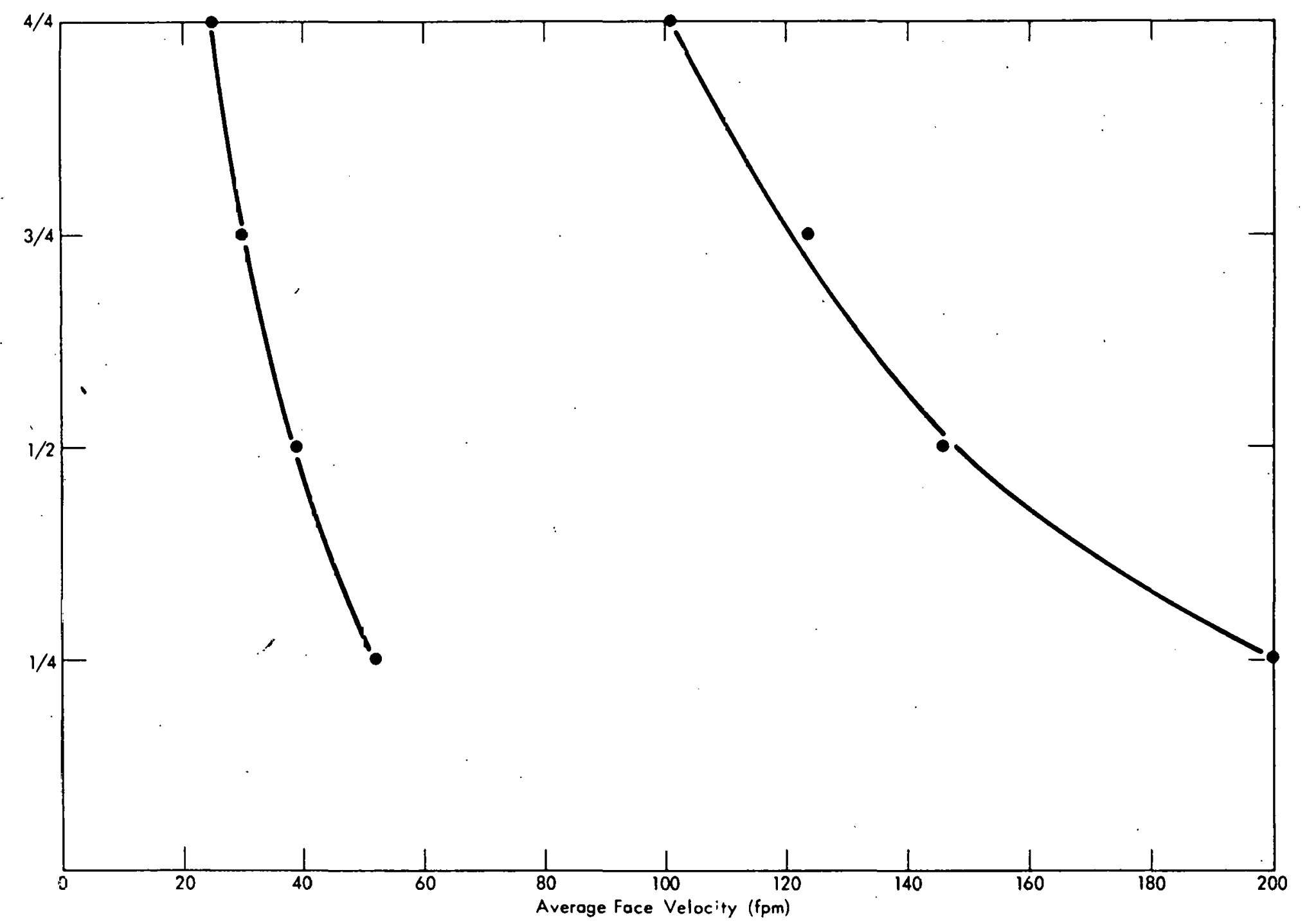

Figure 2. Y-12 Test Hood. 
The inside concentrations were tested 4 inches above the fry pan with a Scott-Davis Halide Meter. Because of turbulence inside the hood, concentrations fluctuated; therefore, meter readings were kept at or slightly above the level necessary for a given concentration. Once a given concentration was establised, 8 readings were taken using the same grid pattern as face velocity readings with a Miran Infrared Gas Analyzer. The top sections corresponded approximately to the breathing zone of one standing at the hood, the bottom sections of one sitting on a high laboratory stool or chair. For each tested material at each inside concentration, 3 tests were made--with the fry pan on the extreme left, in the middle, and in the middle with a 7 inch diameter hot plate turned to its highest setting directly behind it. Tests were to be made at $1,2,5$, and 10 times the threshold limit of the 3 tested vapors. However, if no leakage was detected at the highest concentration further tests were considered unnecessary. In maxginal situations a respirator was worn by the operator to prevent his being exposed to the test chemical. 
Attachment 1

$\mathrm{K}-\mathrm{GD}-826$

\section{CALIBRATION OF LOW VELOCITY ANEMOMETERS}

Several low velocity anemometers were calibrated for environmental and safety personnel at the ORGDP and GAT in a low velocity wind tunnel built specifically for this purpose at the $\mathrm{K}-1303$ Model Test Facility. Calibration was accomplished by video taping heliumfilled soap bubbles as they passed a scale in the test section and calculating velocity by viewing the video tape frame by frame to determine the distance a bubble traveled in a specified time interval.

\section{INSTRUMENTATION}

The setup used in this calibration procedure is shown in Figure 1. The apparatus includes video equipment, a bubble generator, and a low velocity wind tunnel. The video equipment* consists of a highspeed camera, strobe light, recorder, and monitor. The camera takes 60 frames per second and is equipped with a zoom lens. The recorded tape may be played back at actual speed, half-speed, or one frame at a time. The bubble generating equipment at the left side of figure I forms helium-filled neutrally buoyant bubbles of controlled size from $1 / 16$ inch to $1 / 4$ inch diameter. Initially the wind tunnel was a 30-inch by 30-inch duct with a flared inlet and an outlet section converging to the permanent test stand piping. Observation of the flow in this tunnel revealed the presence of large vortices. A honeycomb section 18 inches long and with 2-inch cells was then then added upstream of the test section in conjunction with screens and succeeded in producing an acceptable flow pattern.

\section{PROCEDURE}

The calibration velocity was determined by measuring the distance which a bubble traveled in a specified time interval. A scale was attached inside the test section, and bubbles, introduced through a cell of the honeycomb section, passed about 6 inches in front of the scale. The bubbles passing the scale were video taped with the high-speed camera. The tape of these bubbles was replayed one frame at a time, and individual bubbles which appeared to be flowing parallel to the scale were traced. The distance through which a bubble traveled in a given number of frames, i.e., a specified, accurate time interval, determined its velocity. The average velocity of 20 bubbles was used as the calibration velocity. Only the bubbles which appearcd to travel in a straight line were selected for velocity measurements. The variation of velocities among the individual bubbles was apparent in the turbulence relative intensity $\left(\mathrm{T}_{\mathrm{u}}\right)$.

*

"Videostrobe" Model 800 system. 


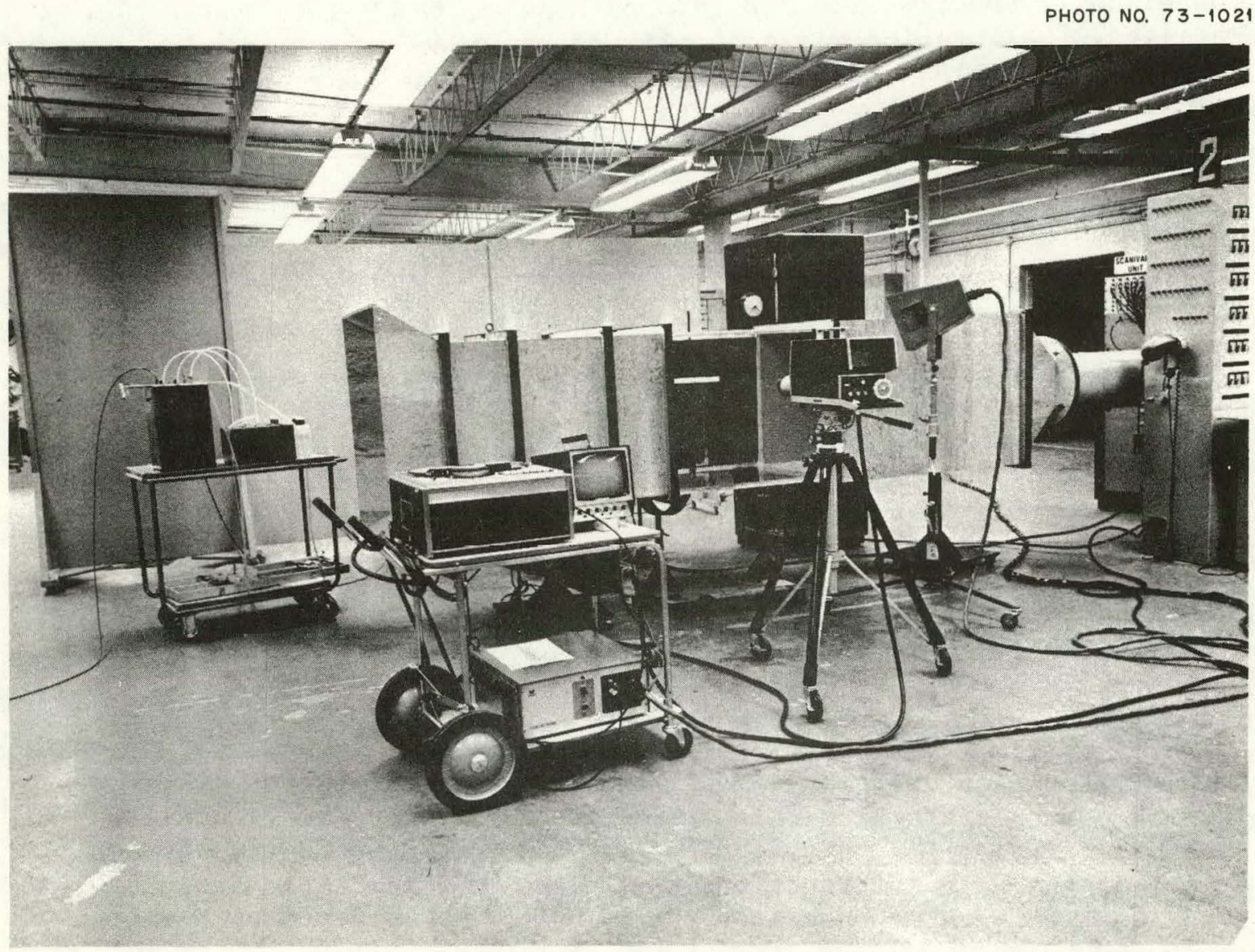

Figure 1. Anemometer Calibration Set-Up. 


$$
T_{u}=\frac{1}{v_{B}}\left[\sum_{i-1}^{n}\left(v_{B}-v_{i}\right)^{2} / n\right]^{1 / 2}
$$

where $\mathrm{n}$ is the number of bubbles used in the calculation;

$\mathrm{v}_{i}$ is the individual bubble velocity; and

$\mathrm{v}_{\mathrm{B}}$ is the average bubble velocity.

\section{RESULTS}

Four consecutive samplings of 20 bubbles showed a mean velocity variation of $2 \%$ and a turbulence intensity variation of $1 \%$. The setup shown in Figure 1 is suitable for velocities from 50 to 800 fpm. For velocities less than $50 \mathrm{fpm}$, the turbulence introduced by the bubble generator probe is detectable. The velocity is limited to $800 \mathrm{fpm}$ by the speed of the camera and the length of the scale.

The instrument to be calibrated was placed inside the test section at the same height as the scale and about 6 inches in front of it. The types of anemometers are shown in Figure 2. The Alnor type 3002 Velometer, the Anemothern 2135, and the Total-Vector anemometer did not function properly and could not be calibrated. The sensitivity of the Fluidic anemometer was so great that proper adjustment could not be maintained in the test section, and, therefore, it could not be calibrated. The remaining Alnor velometer appeared to function reasonably well, but since no operating instructions were available, this instrument may have been improperly used. The two Taylor anemometers, the Alnor Thermo-anemometer, and the Anemothern 2448 functioned properly and were calibrated. The Flow Corporation anemometer was employed mainly to provide a turbulence check.

This low velocity calibration procedure provides an accurate and fairly inexpensive method for anemometer calibration. The system will be maintained for periodic application. 
PHOTO NO. $73-938$

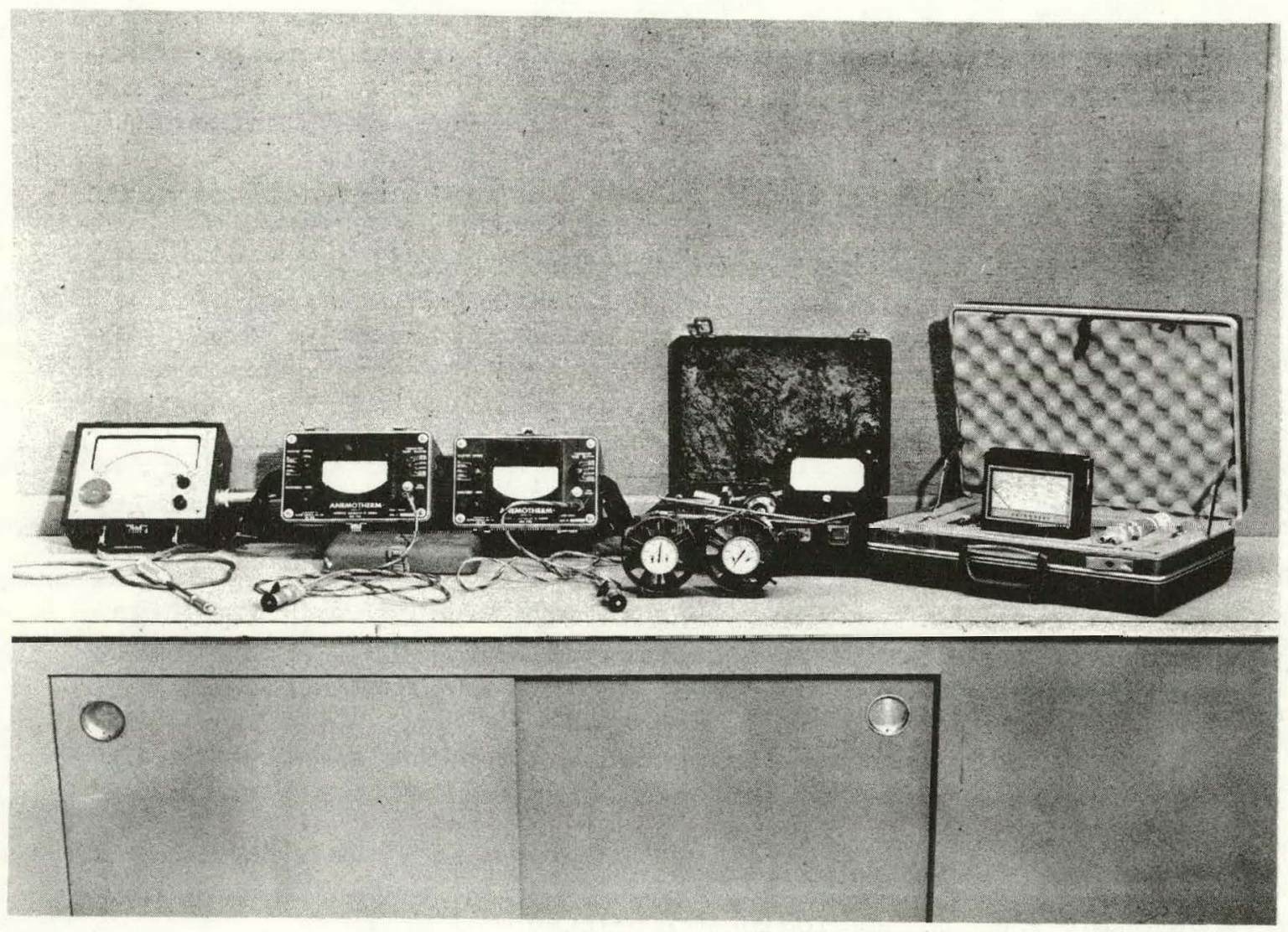

Figure 2. Low Velocity Anemometers. 


\section{INTERNAL DISTRIBUTION}

1. S. P. Alcorn

2. W. S. Everett

3. H. F. Higdon

4-13. H. C. Hoy

14. J. B. Hunt

15. J. F. Morehead

16. W. E. Porter
17-41. Industrial Hygiene Department 42-43. Laboratory Records Department

44. Laboratory Records, ORNL R.C.

45-46. Central Research Library

47. ORNL - Y-12 Technical Library Document Reference Section

48. ORNL Patent Office

EXTERNAL. DISTRIBUTION

49. Director, Research and Technical Support Division, DOE-ORO

50-76. Technical Information Center, P. O. Box 62, Oak Ridge, TN 37830 